\title{
Optimal Capital Taxation and Precautionary Savings
}

Yunmin Chen, YiLi Chien, and C.C. Yang

There are multiple reasons to motivate the role of capital taxation in the heterogenous-agent incomplete-markets (HAIM) model. One is the production inefficiency caused by precautionary savings. The other is the wealth redistribution role played by capital taxation. To distinguish between these two reasons, this article uses an analytical tractable HAIM model with a degenerated distribution of wealth while preserving the role of precautionary savings. The degenerated wealth distribution shuts down the distributional role played by capital taxation. Our results show that, with no role to play for redistribution, the government bond is more suitable than capital tax for addressing the production inefficiency caused by households' precautionary savings in the HAIM model. (JEL C61, E22, E62, $\mathrm{H} 21, \mathrm{H} 30)$

Federal Reserve Bank of St. Louis Review, Third Quarter 2021, 103(3), pp. 333-49. https://doi.org/10.20955/r.103.333-49

\section{INTRODUCTION}

In the Ramsey taxation literature, pivotal work by Chamley (1986) shows that the best way for the government to finance its expenditures in the long run is to tax labor but not capital in a representative-agent model. The recent work by Chari, Nicolini, and Teles (2020) clarifies the conditions and assumptions required to support the zero capital tax result. However, the optimal outcome of this important taxation problem is relatively less understood in the heterogeneous-agent incomplete-markets (HAIM) framework. The HAIM model considers an environment in which households are subject to uninsurable idiosyncratic shocks and ad hoc borrowing restrictions; in response, households buffer their consumption against adverse shocks via precautionary savings. During the past two decades, the HAIM model has become a standard workhorse for policy evaluations in the current state-of-the-art macroeconomics that jointly addresses aggregate and inequality issues. $\frac{1}{}$

Given the importance and popularity of the HAIM model, paralleling the work of Chamley (1986), it is natural to ask, "What is the prescription of Ramsey capital taxation in

Yunmin Chen is an assistant professor at Shandong University. YiLi Chien is a research officer and economist at the Federal Reserve Bank of St. Louis. C.C. Yang is a distinguished research fellow at the Institute of Economics, Academia Sinica.

() 2021, Federal Reserve Bank of St. Louis. The views expressed in this article are those of the author(s) and do not necessarily reflect the views of the Federal Reserve System, the Board of Governors, or the regional Federal Reserve Banks. Articles may be reprinted, reproduced, published, distributed, displayed, and transmitted in their entirety if copyright notice, author name(s), and full citation are included. Abstracts, synopses, and other derivative works may be made only with prior written permission of the Federal Reserve Bank of St. Louis. 


\section{Chen, Chien, Yang}

the long run for the HAIM economy?" There are several important attempts to investigate this question, such as in Aiyagari (1995); Conesa, Kitao, and Krueger (2009); and Dávila et al. (2012). However, some issues still remain unsettled. For example, a possible reason for the positive capital taxation resides in the production efficiency argument. In the equilibrium of the HAIM model, production could be inefficient in the sense that the marginal product of capital is lower than that implied by the so-called "modified golden rule" (MGR hereafter), which is due to the overaccumulation of capital resulting from the precautionary savings of households. However, there are some other reasons to motivate the role of capital taxation in the HAIM economy. For example, in HAIM environments without public debt, the works by Dávila et al. (2012); Krueger and Ludwig (2018); and Conesa, Kitao, and Krueger (2009) suggest that capital taxation or subsidization could also serve to redistribute wealth and thereby improve welfare. This then raises the following question: How do optimal capital taxation and optimal public debt relate to the issue of capital overaccumulation caused by precautionary savings in the HAIM environment?

To answer these questions, we simplify the HAIM model in a particular way so that wealth distribution is degenerated each period. Our article adopts the model of Chen et al. (2021), which follows the work of Lucas (1990) and Heathcote and Perri (2018). More specifically, a special risk-sharing technology is introduced such that individual members within a family are able to pull their wealth by the end of each period. As a result, wealth distribution is totally degenerated each period and hence capital tax can no longer play a role for wealth redistribution. Despite wealth-redistributing technology, our model still preserves income/consumption inequality as well as households' precautionary-saving motives. $\underline{2}$

By isolating the redistributive role of capital tax, our model is therefore suitable for answering the following specific question: Should the government tax capital to achieve the MGR purely because of the production inefficiency caused by precautionary savings? Our results demonstrate that the Ramsey planner intends to increase the supply of government bonds until no individuals are borrowing-constrained in the long run. That is to say, the needs of precautionary savings are fully met by the supply of public debt, and hence capital is no longer overaccumulated. As a result, the long-run optimal capital tax rate becomes zero. In short, with no role to play in redistribution, the government bond is more suitable than capital tax for addressing the production inefficiency caused by precautionary savings in the HAIM model. These results are totally consistent with the recent studies by Chen et al. (2021) and Chien and Wen (2020). However, the later article relies critically on quasi-linear preferences to derive their results.

Our article is related to the earlier work of Aiyagari (1995), which shows that a positive capital tax should be imposed to implement the MGR in an assumed Ramsey steady state. Our zero-capital-taxation result should not be seen as a contradiction to his result, since there is no wealth distribution in our model. Instead, our result helps to clarify that the positive capital tax result in the Aiyagari model is not driven purely by the MGR but is more likely due to the wealth-redistribution role of capital tax.

The remainder of the article is organized as follows: Section 2 describes the model, derives the competitive equilibrium, and provides sufficient conditions for the Ramsey planner to 
support a competitive equilibrium. Section 3 shows how to solve for the Ramsey allocation analytically and how to derive the optimal capital tax in a Ramsey steady state. Section 4 concludes the study.

\section{MODEL}

We adopt the model of Chen et al. (2021), which has the following three major players: firms, government, and households.

\subsection{Environment}

Firms. Time is discrete and the horizon is infinite, indexed by $t=0,1,2, \ldots, \infty$. A representative firm produces output according to the constant returns-to-scale Cobb-Douglas technology, $Y_{t}=F\left(K_{t}, N_{t}\right)=K_{t}^{\alpha} N_{t}^{1-\alpha}$, where $Y, K$, and $N$ denote aggregate output, capital, and labor, respectively. The firm rents capital and hires labor from households by paying a competitive rental rate and real wage, denoted by $q_{t}$ and $w_{t}$, respectively. The firm's optimal conditions for profit maximization at time $t$ satisfy

$$
\begin{gathered}
w_{t}=\frac{\partial F\left(K_{t}, N_{t}\right)}{\partial N_{t}} \text { and } \\
q_{t}=\frac{\partial F\left(K_{t}, N_{t}\right)}{\partial K_{t}} .
\end{gathered}
$$

Government. The government can issue one-period government bonds and levy flatrate, time-varying labor and capital taxes at rates $\tau_{n, t}$ and $\tau_{k, t}$, respectively. The flow government budget constraint at time $t$ is expressed as

$$
\tau_{n, t} w_{t} N_{t}+\tau_{k, t}\left(q_{t}-\delta\right) K_{t}+\frac{B_{t+1}}{R_{t+1}}=B_{t}
$$

where $R_{t+1}$ is the risk-free gross interest rate between time $t$ and $t+1 ; \delta \in(0,1)$ is the depreciation rate of capital; and $B_{t+1}$ is the amount of government bonds issued at time $t$. The government is assumed to fully commit to a sequence of taxes imposed and debts issued, given the initial amount of government bonds $B_{0}$ at time 0 . This setup for the government is standard for the Ramsey problem.

Households. There is a unit measure of ex ante identical individual households that belong to a representative family. Individual households face idiosyncratic employment shocks, denoted by $\theta \in\{e, u\}$. The shocks are identically and independently distributed over time and across all individual households. If $\theta=e$, then individual households can work and receive their labor income. Otherwise, if $\theta=u$, then individual households cannot work and have no labor income. Let $\pi(e)$ and $\pi(u)$ denote the probabilities of employment and unemployment shocks, respectively. There are two subperiods within each period. In the first subperiod, individual households are separated from their representative family and receive the employment 


\section{Chen, Chien, Yang}

shock $\theta$. In addition, their consumption and labor supply decisions (if employed) are made in the first subperiod. In the second subperiod, individual agents are reunited with their representative family and share their assets. Therefore, the idiosyncratic shocks are not fully insurable by their representative family. Given the existence of idiosyncratic risks, individual households still have a precautionary motive to save. However, wealth distribution is degenerated each period since individuals can share their asset holdings in the second subperiod. This model setup is similar to that of Lucas (1990) and Heathcote and Perri (2018).

The lifetime utility of a representative family is assumed to be

$$
U=\sum_{t=0}^{\infty} \beta^{t}\left\{\left[\log c_{t}^{e}+v\left(1-n_{t}^{e}\right)\right] \pi(e)+\left[\log c_{t}^{u}+v(1)\right] \pi(u)\right\},
$$

where $\beta \in(0,1)$ is the discount factor; $c_{t}^{e}$ and $c_{t}^{u}$ denote the consumption for employed and unemployed individuals, respectively, at time $t$; and $n_{t}^{e}$ is the labor supply of employed individuals. Note that the labor supply is zero for unemployed individuals. For simplicity, we work with a logarithmic utility function in consumption and let $v(\cdot)$ satisfy standard assumptions $v^{\prime}>0, v^{\prime \prime}<0$ and $\lim _{n \rightarrow 1} v^{\prime}=\infty$. In addition, the flow budget constraint of the representative family is given by

$$
c_{t}^{e} \pi(e)+c_{t}^{u} \pi(u)+\frac{a_{t+1}}{R_{t+1}} \leq \hat{w}_{t} n_{t}^{e} \pi(e)+a_{t},
$$

where $\hat{w}_{t}=\left(1-\tau_{n, t}\right) w_{t}$ is the after-tax wage rate at time $t$, and $a_{t}$ is the asset holdings. All representative households by assumption have the same initial asset holdings $a_{0}>0$. In addition, there are inter-period budget constraints for employed individuals,

$$
c_{t}^{e} \leq a_{t}+\hat{w}_{t} n_{t}^{e}
$$

and for unemployed individuals,

$$
c_{t}^{u} \leq a_{t}
$$

Finally, government bonds and capital are perfect substitutes for a store of value for individual households. As a result, the after-tax gross rate of return on capital must equal the gross risk-free rate; that is,

$$
R_{t+1}=1+\left(1-\tau_{k, t+1}\right)\left(q_{t+1}-\delta\right)
$$

which constitutes a no-arbitrage condition for capital and bonds.

\subsection{Household Problem}

The representative family chooses a sequence of $\left\{c_{t}^{e}, c_{t}^{u}, n_{t}^{e}, a_{t+1}\right\}_{t=0}^{\infty}$ to maximize (4) subject to (5), (6), and (7). Let $\beta^{t} \lambda_{t}^{e} \pi(e), \beta^{t} \lambda_{t}^{u} \pi(u)$, and $\beta^{t} u_{t}$ denote the Lagrangian multipliers attached to (6), (7), and (5), respectively. The first-order conditions (FOCs) of representative households with respect to $c_{t}^{e}, c_{t}^{u}, n_{t}^{e}$, and $a_{t+1}$ are given by 


$$
\begin{gathered}
\frac{1}{c_{t}^{e}}=\lambda_{t}^{e}+\mu_{t}, \\
\frac{1}{c_{t}^{u}}=\lambda_{t}^{u}+\mu_{t}, \\
v^{\prime}\left(1-n_{t}^{e}\right)=\hat{w}_{t}\left(\lambda_{t}^{e}+\mu_{t}\right), \text { and } \\
\beta\left(\lambda_{t+1}^{e} \pi(e)+\lambda_{t+1}^{u} \pi(u)\right)+\beta \mu_{t+1}=\frac{\mu_{t}}{R_{t+1}},
\end{gathered}
$$

respectively.

\subsection{Competitive Equilibrium}

A competitive equilibrium of the model economy is defined in the standard way.

Definition 1. Given the initial capital $K_{0}$ and initial government bond $B_{0}$, a competitive equilibrium is defined as sequences of tax rates and government bonds $\left\{\tau_{n, t}, \tau_{k, t}, B_{t+1}\right\}$ and sequences of prices $\left\{w_{t}, q_{t}, R_{t}\right\}_{t=0}^{\infty}$, aggregate allocations $\left\{C_{t}, N_{t}, K_{t+1}\right\}_{t=0}^{\infty}$, and individual allocation plans $\left\{c_{t}^{e}, c_{t}^{u}, n_{t}^{e}, a_{t+1}\right\}_{t=0}^{\infty}$, such that the following are true:

(i) Given prices, $\left\{c_{t}^{e}, c_{t}^{u}, n_{t}^{e}, a_{t+1}\right\}$ solves the representative family problem.

(ii) Given prices, $\left\{N_{t}, K_{t}\right\}$ solves the representative firm's problem.

(iii) The no-arbitrage condition, equation (8), holds for all $t$.

(iv) The government budget constraint, equation (3), holds for all $t$.

(v) All markets clear for all $t \geq 0$; that is,

$$
\begin{aligned}
B_{t+1}+R_{t+1} K_{t+1} & =a_{t+1} \\
N_{t} & =n_{t}^{e} \pi(e) \\
C_{t} & =c_{t}^{e} \pi(e)+c_{t}^{u} \pi(u), \text { and } \\
F\left(K_{t}, N_{t}\right) & =C_{t}+K_{t+1}-(1-\delta) K_{t} .
\end{aligned}
$$

\subsection{Characterization of Competitive Equilibrium}

This subsection characterizes several key properties of the competitive equilibrium by means of two propositions.

Proposition 1. The competitive equilibrium of the model has the following two properties:

(i) The variable $c_{t}^{u}$ can be expressed as a function of $c_{t}^{e}$ and $a_{t}$; that is,

$$
c_{t}^{u}=\left\{\begin{array}{l}
c_{t}^{e} \text { if } c_{t}^{e} \leq a_{t} \\
a_{t} \text { if } c_{t}^{e}>a_{t}
\end{array} .\right.
$$


(ii) Equation (12) can be rewritten as

$$
\frac{1}{R_{t+1}}=\beta \frac{\left(1 / c_{t+1}^{e}\right) \pi(e)+\left(1 / c_{t+1}^{u}\right) \pi(u)}{1 / c_{t}^{e}} .
$$

Proof. Please refer to Appendix A1.

Proposition 1 implies that if the asset holdings (or self-insurance position) $a_{t}$ is sufficiently large such that $c_{t}^{e} \leq a_{t}$, then individuals can obtain the same level of consumption regardless of their idiosyncratic shock. We refer to this as a full self-insurance case. Obviously, by the asset market clearing condition (13), this could be achieved if the supply of government bonds is sufficiently high. In addition, if full self-insurance is achieved in the steady state, then the steady-state risk-free rate, $R$, is equal to the time discount rate, $1 / \beta$. Otherwise, $R<1 / \beta$.

Proposition 2. In a laissez-faire competitive equilibrium, the steady-state risk-free rate, $R$, is lower than the time discount rate, $1 / \beta$, if and only if the model parameters satisfy the following condition:

$$
\beta<\frac{1-\alpha}{\alpha+(1-\alpha)(1-\delta)}
$$

Proof. Please refer to Appendix A2.

Proposition 2 provides a sufficient and necessary condition for the risk-free interest rate being lower than the time discount rate in a laissez-faire competitive equilibrium. This is because the idiosyncratic shock is not fully hedged, and hence individual agents' asset holdings may be insufficient to provide full self-insurance for unemployed individuals. In this case, precautionary-saving motives reduce the equilibrium interest rate below the time discount rate. Without government intervention, there is an overaccumulation of capital in the sense that the steady-state capital stock exceeds the level implied by the MGR. This outcome is clearly inefficient in terms of aggregate production from a social point of view. To make our analysis interesting and the Ramsey problem meaningful, we assume that condition (17) holds in this article.

\subsection{Conditions to Support Competitive Equilibrium}

The following proposition demonstrates that the Ramsey planner can pick a competitive equilibrium by choosing aggregate capital plus individual consumption, labor, and asset holdings that satisfy a set of conditions.

Proposition 3. Given the initial capital $K_{0}$, government bonds $B_{0}$, and capital tax rate $\tau_{k, 0}$, sequences of the allocation $\left\{c_{t}^{e}, n_{t}^{e}, c_{t}^{u}, K_{t+1}, a_{t+1}\right\}_{t=0}^{\infty}$ can be supported as a competitive equilibrium if and only if they satisfy the following conditions ${ }^{\underline{3}}$ :

(i) Resource constraints:

$$
F\left(K_{t}, n_{t}^{e} \pi(e)\right)+(1-\delta) K_{t}-c_{t}^{e} \pi(e)-c_{t}^{u} \pi(u)-K_{t+1} \geq 0, \forall t \geq 0 .
$$


(ii) Implementability conditions: $\forall t \geq 0$,

$$
\frac{v^{\prime}\left(1-n_{t}^{e}\right)}{1 / c_{t}^{e}} n_{t}^{e} \pi(e)+a_{t}-c_{t}^{e} \pi(e)-c_{t}^{u} \pi(u)-a_{t+1} \beta \frac{\left(1 / c_{t+1}^{e}\right) \pi(e)+\left(1 / c_{t+1}^{u}\right) \pi(u)}{1 / c_{t}^{e}} \leq 0,
$$

where

$$
c_{t}^{u}=\left\{\begin{array}{l}
c_{t}^{e} \text { if } c_{t}^{e} \leq a_{t} \\
a_{t} \text { if } c_{t}^{e}>a_{t}
\end{array} .\right.
$$

Proof. Please refer to Appendix A3.

Note that given the allocation $\left\{c_{t}^{e}, n_{t}^{e}, c_{t}^{u}, K_{t+1}\right\}_{t=0}^{\infty}$, capital tax rates are chosen according to (8), which can be rewritten as

$$
\tau_{k, t+1}=1-\frac{R_{t+1}-1}{q_{t+1}-\delta}
$$

where the equilibrium $q_{t+1}$ and $R_{t+1}$ are decided by (2) and (16).

\section{RAMSEY OUTCOME}

\subsection{Ramsey Problem}

On the basis of Proposition 3, the Ramsey problem can be represented as maximizing (4) by choosing $\left\{c_{t}^{e}, c_{t}^{u}, n_{t}^{e}, a_{t+1}, K_{t+1}\right\}_{t=0}^{\infty}$ and, subject to conditions (18) and (19) stated in Proposition 3 , given $K_{0}, B_{0}$, and $\tau_{k, 0}$.

Before presenting the Ramsey outcome, we first define the steady state of our HAIM economy.

Definition 2. Given government policies, the steady state of the HAIM economy is defined as a competitive equilibrium where all aggregate and individual quantity variables stay at finite positive values.

As to the Ramsey steady state, it is defined as follows:

Definition 3. The long-run optimal solution to the Ramsey problem is defined as a Ramsey steady state if it features the steady state of the HAIM economy.

\subsection{Ramsey Outcome}

Denote $\beta^{t} \chi_{t}$ and $\beta^{t} \zeta_{t}$ as the Lagrangian multipliers for conditions (18) and (19), respectively. The FOC of the Ramsey problem with respect to $K_{t+1}$ is given by

$$
\chi_{t}=\beta \chi_{t+1}\left(M P_{K, t+1}+1-\delta\right),
$$

where $M P_{K, t+1}$ denotes $\frac{\partial F\left(K_{t+1}, N_{t+1}\right)}{\partial K_{t+1}}$. 


\section{Chen, Chien, Yang}

There are a few important messages here. First, as shown by equation (21), the Ramsey planner prefers to set the level of capital satisfying the MGR in the long run if $\chi_{t}$ converges to a non-zero positive number. This is feasible because the planner can tax capital and/or use government bonds to crowd out capital. If the Ramsey planner chooses to supply enough government bonds such that full self-insurance is achieved (namely, $c_{t}^{e}=c_{t}^{u}$ ) in the long run, then the equilibrium interest rate is equal to the time discount rate as implied by (16). In this case, the Ramsey steady state can achieve the MGR without taxing capital since the overaccumulation of capital is fully crowded out by the issuance of government bonds. Otherwise, capital tax is needed to achieve the MGR.

Second, the availability of government bonds matters critically for equation (21) to be upheld. As shown in Proposition 3, the Ramsey planner can choose any level of aggregate capital stock so long as it satisfies the resource constraint without worrying about the asset market clearing condition (13). This is because the position of government bonds can be adjusted to satisfy the asset market clearing condition. Clearly, without government bonds as a policy tool, equation (21) no longer holds and hence the MGR might not hold even if $\chi_{t}$, the shadow price of the resource constraint, converges.

Finally, the existence of a Ramsey steady state does not require the convergence of Lagrangian multipliers. For example, based on equation (21), a Ramsey steady state could exist if the growth rate of $\chi_{t}$ converges and does not necessarily require the convergence of $\chi_{t}$ itself. Hence, the investigation of the convergence property of these multipliers is important to determine the optimal Ramsey outcome in the long run. This is exactly what we show in the proof of the following proposition, which characterizes the long-run Ramsey outcome. We denote variables without the subscript $t$ as their steady-state values.

Proposition 4. There exist Ramsey steady states, all of which satisfy the following properties:

(i) Full self-insurance is achieved, namely, $a \geq c^{e}=c^{u}$, and the equilibrium interest rate is equal to the time discount rate, $1 / \beta$.

(ii) The MGR holds without taxing capital, $\tau_{k}=0$.

(iii) The optimal level of government bonds is strictly positive if condition (17) is met. The bond interest payments are financed solely by revenues from the labor income tax.

Proof. Please refer to Appendix A4.

There are several key points here. First, in this economy, instead of taxing capital, the Ramsey planner always supplies enough government bonds in the long run to solve the problem of production inefficiency (the failure of the MGR) caused by households' precautionary savings. In other words, capital tax is never used in the Ramsey steady state. Hence, we show that the government bond is a better tool than capital tax to restore production efficiency. This result is fully consistent with Chien and Wen (2020), who show a similar result with a different type of HAIM model.

The intuition behind these results is that issuing government bonds is always a better tool than imposing a capital tax in addressing the capital overaccumulation issue (i.e., production inefficiency issue) caused by precautionary savings in the HAIM model. Issuing government 
bonds can play a role in crowding out overaccumulated capital and improve the self-insurance position among households. In contrast, although imposing a capital tax can alleviate the problem of capital overaccumulation, it does so at the expense of worsening the problem of a lack of full self-insurance caused by incomplete markets.

Second, the interest payment on a government bond is fully financed by the labor tax in the Ramsey steady state. This is true despite the fact that the amount of the interest rate payment could be significant. Our results indicate that it is still optimal to issue debt up to a point where full self-insurance is achieved, even at the cost of a high labor wedge. The intuition underlying our results rests on an intertemporal argument based on the hallmark of the HAIM model, where the risk-free rate is always less than the time discount rate unless full self-insurance is achieved. Unlike individual households faced with an earnings risk, the Ramsey planner in the HAIM economy (without aggregate shocks) faces no uncertainty in allocating aggregate resources. Given that the planner discounts the future by $\beta$, the strict inequality of $R<1 / \beta$ then dictates that the market discounts resources at a lower rate than the planner discounts utility, implying the existence of the planner's desire to improve welfare by front-loading aggregate consumption through issuing more bonds.

Finally, we show that the optimal level of debt is positive only if there is no full self-insurance under the laissez-faire competitive equilibrium. This result again highlights that the purpose in supplying public debt is to satisfy the need for precautionary savings. In other words, with a sufficient amount of public debt, the Ramsey planner can achieve full self-insurance as well as production efficiency.

\section{CONCLUSION}

Our study indicates that the Ramsey planner prefers government bonds to capital tax to fully correct the production inefficiency problem caused by precautionary savings in the HAIM model without wealth distribution. The finding of this article is fully consistent with that of Chen et al. (2021) and Chien and Wen (2020).

This study also improves our understanding and helps to interpret various results found in the Ramsey literature featuring HAIM. There are several important articles that study optimal capital taxation in HAIM models while not considering the role of government debtfor example, Conesa, Kitao, and Krueger (2009); Krueger and Ludwig (2018); and Dávila et al. (2012). In our Ramsey problem, it is easily shown that the MGR does not hold once there are no government bonds. As a result, the optimal capital tax results of these studies do not need to obey the MGR even in the long run. In other words, the availability of government bonds is critical for the Ramsey planner to achieve the MGR. Therefore, our result of zero capital taxation does not contradict the findings of these studies. Hence, the non-zero capital taxation results found by these studies are not motivated by the production-efficient role implied by the MGR, but by the wealth-redistribution role played by capital tax. The latter role is absent from our study for good reason. 


\section{APPENDIX A 1}

\section{Proof of Proposition 1}

By equations (6) and (7), we know that $c_{t}^{u} \leq c_{t}^{e}$ so that $\lambda_{t}^{u} \geq \lambda_{t}^{e}$. Now suppose $\lambda_{t}^{e}>0$. It follows that $\lambda_{t}^{u}>0$. Given $\lambda_{t}^{e}>0$ and $\lambda_{t}^{u}>0$, both (6) and (7) must be binding. Inserting $c_{t}^{e}=a_{t}+\hat{w}_{t} n_{t}^{e}$ and $c_{t}^{u}=a_{t}$ back into (5), we obtain $a_{t+1}=0$, which together with (7) implies that $c_{t+1}^{u}=0$. This leads to a contradiction since $u^{\prime}(0)=\infty$. As a consequence, $\lambda_{t}^{e}$ must be zero. Next, consider two possibilities for $\lambda_{t}^{u}$. One is that $\lambda_{t}^{u}>0$ so that $c_{t}^{u}=a_{t}$ and $c_{t}^{u}<c_{t}^{e}$ (recall that $\lambda_{t}^{e}=0$ ). The other is that $\lambda_{t}^{u}=0$, along with $\lambda_{t}^{u}=\lambda_{t}^{e}=0$, which leads to $c_{t}^{e}=c_{t}^{u} \leq a_{t}$. Hence, we can express $c_{t}^{u}$ as a function of $c_{t}^{e}$ and $a_{t}$ as shown by (15). In addition, using $\lambda_{t}^{e}=0$, we can rewrite (12) as (16).

\section{APPENDIX A2}

\section{Proof of Proposition 2}

Under the laissez-faire competitive equilibrium, $\tau_{n, t}=\tau_{k, t}=B_{t}=0$ such that $a_{t}=R_{t} K_{t}$. Provided $c^{e}>c^{u}=R K$ is true, we can conclude that in the steady state $\beta R<1$. Given this, we first prove that if $c^{e}>c^{u}=R K$, then $\beta<\frac{1-\alpha}{\alpha+(1-\alpha)(1-\delta)}$ must hold.

Based on $R=1+\alpha K^{\alpha-1} N^{1-\alpha}-\delta$, we express $\left(\frac{K}{N}\right)^{\alpha-1}$ as $\frac{R+\delta-1}{\alpha}$. In the steady state, we have Euler equation (16) and the resource constraint,

$$
\begin{aligned}
1 & =\beta R\left(\pi(e)+\frac{c^{e}}{c^{u}} \pi(u)\right), \\
c^{e} \pi(e)+c^{u} \pi(u) & =K^{\alpha} N^{1-\alpha}-\delta K \\
& =\left(\alpha K^{\alpha-1} N^{1-\alpha}-\delta\right) K+(1-\alpha) K^{\alpha-1} N^{1-\alpha} K \\
& =(R-1) K+(1-\alpha)\left(\frac{R+\delta-1}{\alpha}\right) K,
\end{aligned}
$$

where we replace $K^{\alpha-1} N^{1-\alpha}$ with $\frac{R+\delta-1}{\alpha}$ in (23). Dividing each term by $R K$ in (23) gives

$$
\frac{c^{e}}{R K} \pi(e)+\frac{c^{u}}{R K} \pi(u)=\frac{R-1}{R}+\frac{1-\alpha}{\alpha} \frac{R+\delta-1}{R} .
$$

Substituting $c^{u}=R K$ into (22) and (24) yields

$$
\begin{gathered}
\frac{1}{R}-\beta \pi(u) \frac{c^{e}}{R K}-\beta \pi(e)=0 \text { and } \\
\frac{\alpha+(1-\alpha)(1-\delta)}{\alpha} \frac{1}{R}+\frac{c^{e}}{R K} \pi(e)+\pi(u)-\frac{1}{\alpha}=0 .
\end{gathered}
$$


Combining (25) and (26) leads to

$$
f\left(\frac{c^{e}}{R K}\right)=0
$$

where $f: \frac{c^{e}}{R K} \rightarrow \frac{\alpha+(1-\alpha)(1-\delta)}{\alpha} \beta\left(\pi(u) \frac{c^{e}}{R K}+\pi(e)\right)+\frac{c^{e}}{R K} \pi(e)+\pi(u)-\frac{1}{\alpha}$. Since $f^{\prime}>0$ and $f(1)=\frac{\beta(\alpha+(1-\alpha)(1-\delta))+\alpha-1}{\alpha}<0$ by specification, we know that $\frac{c^{e}}{R K}>1$ solves (27).

Next, we prove the condition $\beta<\frac{1-\alpha}{\alpha+(1-\alpha)(1-\delta)}$ guarantees that the steady-state allocations satisfy $c^{e}>c^{u}=R K$ in a laissez-faire competitive equilibrium. Combining (23) and (22) gives

$$
\beta\left(\pi(e)+\frac{c^{e}}{c^{u}} \pi(u)\right)=\frac{1}{R}=\frac{\alpha}{\alpha+(1-\alpha)(1-\delta)}\left(\frac{1}{\alpha}-\frac{C}{R K}\right) .
$$

Given (15), we know that $c^{e}=c^{u}$ if $C \leq R K$ and that $c^{e}>c^{u}$ if $C>R K$. Consider the case $C \leq R K$. Then, from (28) we have

$$
\beta=\frac{\alpha}{\alpha+(1-\alpha)(1-\delta)}\left(\frac{1}{\alpha}-\frac{C}{R K}\right) \geq \frac{1-\alpha}{\alpha+(1-\alpha)(1-\delta)}
$$

where we know $c^{e}=c^{u}$ since $C \leq R K$, and the inequality is due to $\frac{C}{R K} \leq 1$.

It follows that if (29) does not hold, then $C \leq R K$ cannot be true. If $C>R K$ is true, then we have $c^{e}>c^{u}$. Therefore, if $\beta<\frac{1-\alpha}{\alpha+(1-\alpha)(1-\delta)}$, then $C>R K$, and thus $c^{e}>c^{u}$ holds.

\section{APPENDIX A3}

\section{Proof of Proposition 3}

The "If" Part. Given the initial $B_{0}$ and $K_{0}$, as well as the allocation $\left\{c_{t}^{e}, n_{t}^{e}, a_{t+1}, K_{t+1}\right\}_{t=0}^{\infty}$, a competitive equilibrium can be constructed by using the two conditions in Proposition 3 and following the steps below that uniquely back up the sequences of the other variables:

(i) Given $c_{t}^{e}, n_{t}^{e}$, and $a_{t+1}, c_{t}^{u}$ is chosen such that

$$
c_{t}^{u}=\left\{\begin{array}{l}
c_{t}^{e} \text { if } c_{t}^{e} \leq a_{t} \\
a_{t} \text { if } c_{t}^{e}>a_{t}
\end{array} .\right.
$$

Note that $a_{t}-c_{t}^{u} \geq 0$ is satisfied.

(ii) Aggregate consumption and labor, $C_{t}$ and $N_{t}$, are chosen such that

$$
\begin{aligned}
& N_{t}=n_{t}^{e} \pi_{e} \\
& C_{t}=c_{t}^{e} \pi^{e}+c_{t}^{e} \pi^{e} .
\end{aligned}
$$


(iii) Wage rate and capital rental rate, $w_{t}$ and $q_{t}$, are chosen such that $w_{t}=F_{L}\left(K_{t}, N_{t}\right)$ and $q_{t}=F_{K}\left(K_{t}, N_{t}\right)$. As a result, the firm's problem is solved.

(iv) The labor tax rate, $\tau_{n, t}$, is chosen such that $\hat{w}_{t}=\left(1-\tau_{n, t}\right) w_{t}$ satisfies $v^{\prime}\left(1-n_{t}^{e}\right)=\frac{\hat{w}_{t}}{c_{t}^{e}}$.

(v) The risk-free rate, $R_{t+1}$, is chosen by the household Euler equation,

$$
\frac{1}{R_{t+1}}=\beta \frac{\left(1 / c_{t+1}^{e}\right) \pi(e)+\left(1 / c_{t+1}^{u}\right) \pi(u)}{1 / c_{t}^{e}}
$$

and $\tau_{k, t+1}$ is decided by

$$
R_{t+1}=1+\left(1-\tau_{k, t+1}\right)\left(q_{t+1}-\delta\right)
$$

(vi) Given $K_{t+1}$ and $a_{t+1}, B_{t+1}$ is pinned down by the asset market clearing condition

$$
B_{t+1}+R_{t+1} K_{t+1}=a_{t+1}
$$

(vii) There are only two conditions left-the resource constraint and the representative household's budget constraint. Both are listed in Proposition 3. The implementability condition (derived from the representative household's budget constraint) can be expressed as

$$
\frac{v^{\prime}\left(1-n_{t}^{e}\right)}{1 / c_{t}^{e}} n_{t}^{e} \pi(e)+a_{t}-c_{t}^{e} \pi(e)-c_{t}^{u} \pi(u)-a_{t+1} \beta \frac{\left(1 / c_{t+1}^{e}\right) \pi(e)+\left(1 / c_{t+1}^{u}\right) \pi(u)}{1 / c_{t}^{e}} \leq 0 .
$$

The "Only If" Part. The constraints listed in Proposition 3 are trivially satisfied because they are part of the competitive-equilibrium conditions.

\section{APPENDIX A4}

\section{Proof of Proposition 4}

First, the FOC with respect to $n_{t}^{e}$ is given by

$$
-v^{\prime}\left(1-n_{t}^{e}\right)+\chi_{t} F_{N, t}-\zeta_{t}\left[\frac{v^{\prime}\left(1-n_{t}^{e}\right)}{1 / c_{t}^{e}}-\frac{v^{\prime \prime}\left(1-n_{t}^{e}\right)}{1 / c_{t}^{e}} n_{t}^{e}\right]=0,
$$

which implies that the growth rates of $\chi_{t}$ and $\zeta_{t}$ in the steady state are the same. To see this, rewrite (30) as

$$
\chi_{t} \frac{F_{N, t}}{v^{\prime}\left(1-n_{t}^{e}\right)}-\zeta_{t} \frac{1+1 / \eta_{t}}{1 / c_{t}^{e}}=1
$$

where $\eta_{t}$ is defined as the Frisch labor supply elasticity, $\left.\eta_{t} \equiv \frac{\hat{w}}{n^{e}} \frac{d n^{e}}{d \hat{w}}\right|_{u_{c}}=-\frac{v^{\prime}\left(1-n_{t}^{e}\right)}{v^{\prime \prime}\left(1-n_{t}^{e}\right) n_{t}^{e}}$. 
Hence, in the steady state it must be the case where $\chi_{t}$ and $\zeta_{t}$ grow at the same rate as the Ramsey steady state.

In what follows, we show that there exists a Ramsey steady state exhibiting full selfinsurance and that there is no Ramsey steady state with $R<1 / \beta$. We discuss the following three cases: (i) $a_{t}>c_{t}^{e}=c_{t}^{u}$, (ii) $a_{t}=c_{t}^{e}=c_{t}^{u}$, and (iii) $c_{t}^{e}>c_{t}^{u}=a_{t}$.

(i) Consider the case where $a_{t}>c_{t}^{e}$, implying that $c_{t}^{e}=c_{t}^{u}=c_{t}$. The FOCs with respect to $a_{t+1}$ and $c_{t}$ satisfy

$$
\begin{gathered}
a_{t+1}: \zeta_{t+1}=\zeta_{t} \frac{c_{t}^{e}}{c_{t+1}^{e}} \text { and } \\
c: \frac{1}{c_{t}}-\chi_{t}+\zeta_{t}-a_{t} \zeta_{t-1} \frac{c_{t-1}^{e}}{\left(c_{t}^{e}\right)^{2}}+a_{t+1} \beta \zeta_{t}\left(\frac{\pi(e)}{c_{t+1}^{e}}+\frac{\pi(u)}{c_{t+1}^{u}}\right)-\zeta_{t} v^{\prime}\left(1-n_{t}^{e}\right) n_{t}^{e} \pi(e)=0 .
\end{gathered}
$$

In the Ramsey steady state, we have $\zeta_{t+1}=\zeta_{t}=\zeta$ and $\chi_{t+1}=\chi_{t}=\chi$, according to (30) and (31). Given this, (32) becomes

$$
\frac{1}{c^{e}}-\chi+\zeta\left(1-\frac{a}{c^{e}}(1-\beta)-\frac{\hat{w}}{c^{e}} n^{e} \pi(e)\right)=0
$$

where $\frac{\hat{w}}{c^{e}}=v^{\prime}\left(1-n^{e}\right)$.

Given that $c^{e}=c^{u}$, we know that $\beta R=1$ in the steady state. With $\beta R=1$, we can rewrite the budget constraint as

$$
\widehat{w} n^{e} \pi(e)+a(1-\beta)-c=0 .
$$

Combining (33) and (34) leads to

$$
\frac{1}{c^{e}}=\frac{1}{c^{u}}=\chi .
$$

Since in the Ramsey steady state $\chi_{t+1}=\chi_{t}=\chi$, we know that the MGR holds; that is, $\beta\left(M P_{K}+1-\delta\right)=1$. Thus we obtain $\tau_{k}=0$ in the Ramsey steady state.

(ii) We then consider the case where $a_{t}=c_{t}^{e}=c_{t}^{u}$ and the FOC with respect to $c_{t}^{e}$ satisfies

$$
\begin{aligned}
& 0=\beta^{t} \frac{1}{a_{t}}-\beta^{t} \chi_{t}+\beta^{t-1} \zeta_{t-1} \beta\left[\left(c_{t-1}^{e} / c_{t}^{e}\right) \pi(e)+\left(c_{t-1}^{e} / c_{t}^{u}\right) \pi(u)\right]-\beta^{t} \zeta_{t} \\
& +\beta^{t} \zeta_{t} a_{t+1} \beta\left[\frac{\pi(e)}{c_{t+1}^{e}}+\frac{\pi(u)}{c_{t+1}^{u}}\right]+\beta^{t} \zeta_{t} \\
& -\beta^{t} \zeta_{t} v^{\prime}\left(1-n_{t}^{e}\right) n_{t}^{e} \pi(e)-\beta^{t-1} \zeta_{t-1} a_{t} \beta\left[\frac{c_{t-1}^{e} \pi(e)}{c_{t}^{e} c_{t}^{e}}+\frac{c_{t-1}^{e} \pi(u)}{c_{t}^{u} c_{t}^{u}}\right]
\end{aligned}
$$

The steady-state version of the above equation and implementability condition evaluated at $a_{t}=c^{e}=c^{u}$ are 


$$
\begin{gathered}
\chi_{t}=\frac{1}{a}+\zeta_{t}\left(\beta-v^{\prime}\left(1-n^{e}\right) n^{e} \pi(e)\right) \text { and } \\
v^{\prime}\left(1-n^{e}\right) n^{e} \pi(e)=\beta .
\end{gathered}
$$

It follows that

$$
\chi_{t}=\frac{1}{a} .
$$

As a result, $\chi_{t}$ converges, implying that the MGR holds and that $\tau_{k}=0$.

(iii) Finally, we show there is no Ramsey steady state featuring $R<1 / \beta$. In this case, $c_{t}^{e}>c_{t}^{u}=a_{t}$. The FOCs with respect to $c_{t}^{e}$ and $c_{t}^{u}$ satisfy

$$
\begin{aligned}
& c_{t}^{e}: \frac{\pi(e)}{c_{t}^{e}}-\chi_{t} \pi(e)-\zeta_{t} v^{\prime}\left(1-n_{t}^{e}\right) n_{t}^{e} \pi(e)+\zeta_{t} \pi(e) \\
& +\zeta_{t} a_{t+1} \beta\left[\left(1 / c_{t+1}^{e}\right) \pi(e)+\left(1 / c_{t+1}^{u}\right) \pi(u)\right]-\zeta_{t-1} a_{t} \frac{c_{t-1}^{e}}{c_{t}^{e} c_{t}^{e}} \pi(e) \\
& =0 \text { and } \\
& c_{t}^{u}: \frac{\pi(u)}{c_{t}^{u}}-\chi_{t} \pi(u)+\zeta_{t} \pi(u)-\zeta_{t} \\
& +\zeta_{t-1}\left[\left(c_{t-1}^{e} / c_{t}^{e}\right) \pi(e)+\left(c_{t-1}^{e} / c_{t}^{u}\right) \pi(u)\right]-\zeta_{t-1} a_{t} \frac{c_{t-1}^{e}}{c_{t}^{u} c_{t}^{u}} \pi(u) \\
& =0 .
\end{aligned}
$$

By the implementability condition, we rewrite (35) as

$$
\frac{\pi(e)}{c_{t}^{e}}-\chi_{t} \pi(e)+\zeta_{t} \frac{c_{t}^{u}}{c_{t}^{e}} \pi(e)-\zeta_{t-1} c_{t}^{u} \frac{c_{t-1}^{e}}{c_{t}^{e} c_{t}^{e}} \pi(e)=0 .
$$

Rearranging (36) and (37) and then evaluating them in the steady state, we obtain

$$
\begin{aligned}
& \frac{\pi(u)}{c^{u}}-\chi_{t} \pi(u)=\left(\zeta_{t}-\zeta_{t-1}\right) \pi(e) \text { and } \\
& \frac{\pi(e)}{c^{e}}-\chi_{t} \pi(e)=\left(\zeta_{t-1}-\zeta_{t}\right) \frac{c^{u}}{c^{e}} \pi(e) .
\end{aligned}
$$

Let $g$ denote the growth rate of $\chi_{t}$ as well as $\zeta_{t}$. (Recall that $\chi_{t}$ and $\zeta_{t}$ must grow at the same rate in the steady state.) Given $\frac{1}{c^{u}}>\frac{1}{c^{e}}$, from (38) and (39) we obtain $\left(\zeta_{t}-\zeta_{t-1}\right) \frac{\pi(e)}{\pi(u)}>\left(\zeta_{t-1}-\zeta_{t}\right) \frac{c^{u}}{c^{e}}$, expressed as

$$
\left(\zeta_{t}-\zeta_{t-1}\right) \frac{\pi(e)}{\pi(u)}-\left(\zeta_{t-1}-\zeta_{t}\right) \frac{c^{u}}{c^{e}}=\left(\zeta_{t}-\zeta_{t-1}\right)\left(\frac{\pi(e)}{\pi(u)}+\frac{c^{u}}{c^{e}}\right)>0,
$$


which implies that $g \equiv \frac{\zeta_{t}}{\zeta_{t-1}}>1$. Manipulating (38) with $\frac{\pi(u)}{c_{t}^{u}}=\frac{\pi(u)}{c_{t-1}^{u}}$ gives

$$
\chi_{t} \pi(u)+\left(\zeta_{t}-\zeta_{t-1}\right) \pi(e)=\chi_{t-1} \pi(u)+\left(\zeta_{t-1}-\zeta_{t-2}\right) \pi(e) .
$$

Given $\chi_{t}=g \chi_{t-1}, \zeta_{t}=g \zeta_{t-1}$, and $\zeta_{t-2}=\frac{1}{g} \zeta_{t-1}$, we can rewrite the above equation as

$$
g \chi_{t-1} \pi(u)+\left(g \zeta_{t-1}-\zeta_{t-1}\right) \pi(e)=\chi_{t-1} \pi(u)+\left(\zeta_{t-1}-\frac{1}{g} \zeta_{t-1}\right) \pi(e)
$$

Hence, we have

$$
\chi_{t-1} \pi(u)=\pi(e) \zeta_{t-1}\left(\frac{1-g}{g}\right)
$$

If $\zeta_{t-1}>0$, (40) implies that $\chi_{t-1}<0$, which leads to a contradiction, given that both $\zeta_{t-1}$ and $\chi_{t-1}$ must be non-negative. If $\zeta_{t-1}=0$, then $\chi_{t-1}=0$ and $\zeta_{t-1}=0$. We rule out $\zeta_{t-1}=\chi_{t-1}=0$ because $c^{e}=c^{u} \rightarrow \infty$, obtained by (38) and (39), is impossible.

Finally, we show that the optimal level of debt is strictly positive if condition (17) is met. Let us consider the lowest amount of government debt that achieves full self-insurance. That is, consider the steady state case where $a=c^{e}=c^{u}$.

By the flow government budget constraint in the Ramsey steady state, the steady-state labor tax rate can be written as a function of the debt-to-GDP ratio, $B / Y$,

$$
\tau_{n}=(1-\beta) \frac{B}{Y} \frac{1}{\frac{w N}{Y}}=\frac{(1-\beta)}{(1-\alpha)} \frac{B}{Y},
$$

where the last equality uses the labor share $\frac{w N}{Y}=1-\alpha$. In addition, the steady-state version of the household budget constraint implies that the asset-holding-to-GDP ratio is given by

$$
\frac{a}{Y}=\left(1-\tau_{n}\right) \frac{w N}{Y} \frac{1}{\beta}=\left(1-\tau_{n}\right) \frac{1-\alpha}{\beta} .
$$

In addition, we know that the MGR holds in this Ramsey steady state, which gives

$$
\frac{K}{Y}=\frac{\alpha}{\frac{1}{\beta}-1+\delta}
$$

By plugging the three equations above together into the asset market clearing condition, we can obtain

$$
\frac{B}{Y}=\frac{a}{Y}-\frac{1}{\beta} \frac{K}{Y}=\left(1-\frac{(1-\beta)}{(1-\alpha)} \frac{B}{Y}\right) \frac{1-\alpha}{\beta}-\frac{1}{\beta} \frac{K}{Y},
$$

which can solve the steady-state $B / Y$ ratio as 


\section{Chen, Chien, Yang}

$$
\frac{B}{Y}=1-\alpha-\frac{\alpha}{\frac{1}{\beta}-1+\delta}
$$

Hence, the optimal debt is positive if $1-\alpha>\frac{\alpha}{\frac{1}{\beta}-1+\delta}$, which is exactly condition (17).

\section{NOTES}

1 It is also known as the Bewley-Huggett-Aiyagari model. For surveys of the literature, see Heathcote, Storesletten, and Violante (2009); Guvenen (2011); Ljungqvist and Sargent (2012); Quadrini and Ríos-Rull (2015); and Krueger, Mitman, and Perri (2016).

$\underline{2}$ This is assuming the quasi-linear utility is an alternative approach to simplifying the heterogenous-agent models. For example, see Wen (2009), Challe and Ragot (2011), Wen (2015), and Chien and Wen (2020).

3 The initial capital tax rate, $\tau_{k, 0}$, should be a choice variable for the Ramsey planner. However, given that the initial capital is pre-installed and that households are homogeneous at time zero, taxing the initial capital is essentially the same as allowing a lump-sum tax. As is standard in the literature, we restrict the planner's ability to choose $\tau_{k, 0}$ in the Ramsey problem.

\section{REFERENCES}

Aiyagari, S.R. "Optimal Capital Income Taxation with Incomplete Markets, Borrowing Constraints, and Constant Discounting." Journal of Political Economy, 1995, 103(6), pp. 1158-75; https://doi.org/10.1086/601445.

Challe, E. and Ragot, X. "Fiscal Policy in a Tractable Liquidity-Constrained Economy." Economic Journal, 2011, 121(551), pp. 273-317; https://doi.org/10.1111/j.1468-0297.2010.02399.x.

Chamley, C. "Optimal Taxation of Capital Income in General Equilibrium with Infinite Lives." Econometrica, 1986, 54(3), pp. 607-22; https://doi.org/10.2307/1911310.

Chari, V.; Nicolini, J.P. and Teles, P. “Optimal Capital Taxation Revisited.” Journal of Monetary Economics, 2020, 116(C), pp. 147-65; https://doi.org/10.1016/j.jmoneco.2019.09.015.

Chen, Y.; Chien, Y.; Wen, Y. and Yang, C. "Should Capital Be Taxed?" Economics Letters, 2021, Volume 200; https://doi.org/10.1016/j.econlet.2021.109746.

Chien, Y. and Wen, Y. “Don't Tax Capital-Optimal Ramsey Taxation in Heterogeneous Agent Economies with QuasiLinear Preferences." Working Paper No. 2019-007D, Federal Reserve Bank of St. Louis, May 2020; https://doi.org/10.20955/wp.2019.007.

Conesa, J.C.; Kitao, S. and Krueger, D. "Taxing Capital? Not a Bad Idea after All!" American Economic Review, 2009, 99(1), pp. 25-48; https://doi.org/10.1257/aer.99.1.25.

Dávila, J.; Hong, J.H.; Krusell, P. and Ríos-Rull, J.-V. “Constrained Efficiency in the Neoclassical Growth Model with Uninsurable Idiosyncratic Shocks." Econometrica, 2012, 80(6), pp. 2431-67; https://doi.org/10.3982/ECTA5989.

Guvenen, F. “Macroeconomics with Heterogeneity: A Practical Guide.” Economic Quarterly, 2011, 97(3), pp. 255-326; https://doi.org/10.3386/w17622.

Heathcote, J. and Perri, F. "Wealth and Volatility." Review of Economic Studies, 2018, 85(4), pp. 2173-213; https://doi.org/10.1093/restud/rdx074.

Heathcote, J.; Storesletten, K. and Violante, G.L. "Quantitative Macroeconomics with Heterogeneous Households." Annual Review of Economics, 2009, 1(1), pp. 319-54; https://doi.org/10.1146/annurev.economics.050708.142922. 
Krueger, D. and Ludwig, A. "Optimal Taxes on Capital in the OLG Model with Uninsurable Idiosyncratic Income Risk." NBER Working Paper No. 24335, National Bureau of Economic Research, 2018; https://doi.org/10.3386/w24335.

Krueger, D.; Mitman, K. and Perri, F. "Macroeconomics and Household Heterogeneity," in, J.B. Taylor and H. Uhlig, eds. Handbook of Macroeconomics. Vol. 2. Amsterdam: North Holland, 2016, pp. 843-921; https://doi.org/10.1016/bs.hesmac.2016.04.003.

Ljungqvist, L. and Sargent, T.J. Recursive Macroeconomic Theory. Third Edition. The MIT Press, 2012.

Lucas, R. “Liquidity and Interest Rates." Journal of Economic Theory, 1990, 50(2), pp. 237-64; https://doi.org/10.1016/0022-0531(90)90001-Z.

Quadrini, V. and Ríos-Rull, J.-V. "Inequality in Macroeconomics," in A. Atkinson and F. Bourguignon, eds. Handbook of Income Distribution. Vol. 2. Amsterdam: North-Holland, 2015, pp. 1229-302; https://doi.org/10.1016/B978-0-444-59429-7.00015-7.

Wen, Y. "An Analytical Approach to Buffer-Stock Saving." Working Paper No. 2009-026A, Federal Reserve Bank of St. Louis, July 2009; https://doi.org/10.20955/wp.2009.026.

Wen, Y. "Money, Liquidity and Welfare." European Economic Review, 2015, 76(C), pp. 1-24; https://doi.org/10.1016/j.euroecorev.2015.01.012. 
